\title{
Urgences
}

\section{Du lieu extérieur au lieu intérieur}

\section{René Derouin}

Numéro 17-18, octobre 1987

L'esprit des lieux

URI : https://id.erudit.org/iderudit/025419ar

DOI : https://doi.org/10.7202/025419ar

Aller au sommaire du numéro

Éditeur(s)

Urgences

ISSN

0226-9554 (imprimé)

1927-3924 (numérique)

Découvrir la revue

Citer ce document

Derouin, R. (1987). Du lieu extérieur au lieu intérieur. Urgences, (17-18), 43-54. https://doi.org/10.7202/025419ar

Ce document est protégé par la loi sur le droit d'auteur. L’utilisation des services d'Érudit (y compris la reproduction) est assujettie à sa politique d'utilisation que vous pouvez consulter en ligne.

https://apropos.erudit.org/fr/usagers/politique-dutilisation/
Cet article est diffusé et préservé par Érudit.

Érudit est un consortium interuniversitaire sans but lucratif composé de l’Université de Montréal, l’Université Laval et l’Université du Québec à Montréal. Il a pour mission la promotion et la valorisation de la recherche. https://www.erudit.org/fr/ 


\section{René Derouin \\ DU LIEU EXTÉRIEUR AU LIEU INTÉRIEUR}

J'ai beaucoup de visuel à vous montrer. J'ai des diapositives et deux vidéos.

C'est difficile de parler après Frédéric-Jacques Temple, c'était très beau. Et après la visite de ce matin au Bic, dans un vrai lieu.

Je suis un peu mal à l'aise de vous présenter uniquement des images d'autres lieux où moi j’ai vécu.

Je pense que je vais essayer de vous faire passer un lieu strictement imaginaire, qui va être un lieu de communication de mon cheminement.

Dans les diapositives, je vais faire un résumé de mes trente dernières années: du Mexique en 1955, quand j'ai commencé à faire de la gravure, à la nordicité en 1979, et au-delà. Ensuite, ce sera un vidéo que j'intitule Le lieu extérieur. Je regrette que le vidéo soit en langue anglaise mais c'est un réalisateur de Toronto qui a considéré que mon lieu était important et qui est venu faire un film chez moi. Cela parle de la construction de la maison, de Val-David comme village et comme lieu de travail. Puis je terminerai avec un autre vidéo (sonore - on a fait les percussions à partir de la presse avec laquelle j'imprime -, 8 minutes) que j'ai réalisé l'hiver dernier avec une équipe de Val-David et qui s'intitule Échographie de la mémoire génétique: une murale de 1936 modulés de mémoire, effacée au jet de sable. C'est la première fois que je le présente. Il est très près de l'exposition dont le vernissage aura lieu demain soir.

\section{Donc, les diapositives.}

Voici une aquarelle que j'ai faite en 1955 dans un parc à Mexico. J'ai toujours été influencé dans mon travail par les lieux. Je vais sûrement être influencé par Rimouski. Ca fait une semaine que je suis ici, et je pense que je ne suis pas ailleurs qu'à Rimouski. En 1955, j'étais donc à Mexico. Et j'étais à Mexico peut-être à la recherche d'un lieu que je n'avais pas trouvé ici. C'est au Mexique que j'ai commencé à faire mon cheminement en gravure. La première linogravure que j'ai faite cette année-là, c'est un marché mexicain. J'ai tellement d'affinités avec le Mexique que c'est peut-être là que j'ai trouvé mon identité, mais mon identité nordique. C'est par le contraire que j'ai trouvé qui j'étais, parce que les Mexicains m'ont interrogé là dessus quand j'avais 18 et 19 an: en 1955 . Je ne savais pas alors qui j'étais, et je pense que beaucoup de gens ici au pays ne savaient pas qui ils étaient. 
Quand je suis revenu du Mexique, j'ai décidé d'inventorier mon lieu. J'ai entrepris la réalisation d'une série de gravures sur bois que j'ai intitulée Hiver. Ce n'était sûrement pas dans la mode de l'art de ces années-là, mais c'était mon environnement le plus immédiat. C'était essentiellement basé sur des paysages, sur les rochers, le vent, l'hiver, la lumière et les motoneiges (très populaires dans les années 1965-1966).

Voici une série de gravures sur bois inspirées des techniques de la gravure sur bois japonaise. Elle a été réalisée avant mon séjour à Tokyo, en 1968.

En 1969, j'ai réalisé un livre de 10 gravures qui s'intitule DeadLine. C'est beaucoup relié à un lieu. J'habitais alors dans une vieille maison de pierre le long du Saint-Laurent, dans un lieu en grande transformation parce que dans la région de Montréal, un lieu menacé par les industries pétrochimiques et par l'industrialisation rurale. J'ai donc fait ces gravures qui sont entre les nouvelles technologies (qui s'en viennent) et le paysage (qui disparaît). On voit dans l'ensemble du livre les deux types d'images: c'est vraiment deux sortes de monde.

J'appréhendais aussi, peut-être, la société informatisée et, plutôt que de la vivre en images, je me suis dit: “je vais partir». J'ai décidé de partir pour les États-Unis, et je suis allé travailler en Californie. J'ai alors complètement abandonné le paysage. J'ai réalisé une vingtaine de gravures sur la haute technologie, l'informatique et les centrales nucléaires. Ces gravures datent des années 1970. J'essayais de visiter là-bas tout ce qui était une espèce de prospective du futur. J'ai vraiment inventorié la Californie qu'on imagine actuellement. Silicone Valley. Pas la Californie de la mer et des rives telle qu'on la connaissait à l'époque.

En 1975, j'ai trouvé un lieu. Je l'ai vraiment trouvé dans les Laurentides. Après les itinérances que j'avais faites au Mexique, aux États-Unis et au Japon pendant vingt ans, j'ai décidé de m'installer quelque part.

De la construction d'une maison est sortie une oeuvre qui s'intitule Précambrien. Suite nordique et Empreintes et reliefs, que j'appelle Les lieux extérieurs. Est-il possible de parler de l'esprit des lieux intérieurs sans avoir trouvé son lieu extérieur? Ma quête d'identité nord/sud, était-ce pour me faire une surface, un corps, un enracinement? C'est le minimum vital d'avoir un lieu extérieur. Et c'est vraiment un problème d'identité que je vivais. Je crois que l'ensemble des Québécois l'ont vécu. 
Après que cette maison a été construite, j'ai commencé à inventorier l'ensemble du territoire. C'était très près de la cartographie. L'idée de ces murales en gravures sur bois vient de la carte routière ou de la carte qu'on déplie sous la forme modulaire. C'est comme si le territoire, à partir de la maison, je l'agrandissais. J'ai donc commencé à l'inventorier par des voyages de pêche dans les Hautes Laurentides et ensuite dans le Grand Nord, dans la région de la Baie James.

Voici le parc La Vérendrye. Je regardais le paysage du haut des airs, par avion ou par hélicoptère. C'est comme si le territoire s'agrandissait tout le temps. J'en prenais possession et je le nommais. Les géographes et les cartographes vont se reconnaître bien que ce soit figuratif en ce sens que ce ne sont pas des vues tirées de photographies mais d'observations faites sur place, comme des vues par satellite.

Je reviens à la maison. C'est une maison qui n'a jamais été habitée par d'autres, qui a été dessinée par moi et conçue en fonction du travail que je voulais réaliser. Cela a modifié entièrement mon oeuvre. J'ai pu commencer à réaliser des murales et mettre en application le territoire psychique que j'avais perçu déjà, que j'imaginais.

Cette première murale en gravure sur bois est faite sur la forme modulaire et elle est d'une démesure... à l'image du territoire nordique. Elle s'intitule Le Nouveau-Québec et comprend vingt et un modules. J'ai mis un an à la réaliser, ce qui est à peu près le même effort, en terme de performance technique, que de construire la maison.

Ce qui était peut-être le plus difficile dans la réalisation de cette murale, c'était de maintenir, durant une année entière, un premier souffle, et de garder l'énergie, de garder le mouvement et la spontanéité dans une technique qui est tout à fait traditionnelle. Faire de la gravure sur bois, c'est très simple, mais il reste qu'il faut le faire et qu'il faut maintenir le geste spontané sur une très longue période. Au bout de six mois, j'ai failli lâcher parce que c'était très laborieux.

Voici des modules de cette murale du Nouveau-Québec, modules qui sont inspirés des sources d'énergie du Nord (les grandes rivières, les tourbières) et qui, sur le plan de la couleur, ont sûrement des influences avec le Mexique.

Une fois que je l'ai eue terminée, j'ai repris l'image que j'avais 
créée et je l'ai remise dans le paysage qui est à côté de la maison, car mon atelier n'est pas assez grand pour voir l'ensemble. Voilà le paysage dans le paysage, la murale dans tout ce qu'elle est! C'était la première fois que je pouvais voir l'ensemble du territoire, que je le regardais. Elle était vraiment conforme, dans toute la géologie du sol, du précambrien, à l'observation que j'avais faite.

Ces oeuvres-là ont été exposées au Musée d'art contemporain, à Montréal, en 1981. C'est là que j'ai mis en corrélation les gravures et les plaques. Je voulais tellement - il y a toujours un côté didactique dans la communication de mon oeuvre - que les gens voient le paysage du haut des airs. J'ai donc mis les grandes plaques de bois au plancher: les gens pouvaient faire le tour de ces plaques et regarder le paysage exactement comme moi je l'avais vu. Les plaques en relief étaient donc, en partie, exposées au plancher.

Ici, c'est une autre partie de l'exposition intitulée Empreintes et reliefs. Â droite, la gravure intitulée Taïga (la taïga est cette partie du territoire de la forêt boréale qui est avant la toundra) et, à gauche, la plaque qui a servi àl'impression. Au plancher, enfin, l'empreinte, dans du sable de fonderie. C'était une première application du thème Between.

Quand on se promenait au musée, on voyait l'empreinte et le relief. On imaginait les reliefs de la croûte terrestre, puis on voyait la plaque. Cela s'est modifié encore par la suite. C'est une étape importante dans mon travail parce que je me suis retrouvé entre deux choses: entre la plaque et l'impression. Je me suis dit: «j'abandonne la gravure et je vais faire uniquement des plaques en relief (basrelief)».

Ceci est l'exposition Suite nordica (1985-1986) dans le contraire de la nordicité, à Mexico, au Musée E1 Chopo, trente ans après la découverte en quelque sorte de mon identité dans cette ville, où j'ai apporté "qui j'étais?", "quel territoire j'habitais?» et «d'où je venais?», toutes les questions que les Mexicains me posaient en 1955 et auxquelles je ne pouvais pas répondre.

Ceci est une première gravure de la série intitulée Between. Une pièce unique (environ 6 pieds $\times 8$ pieds): à droite, la plaque et, à gauche, la gravure imprimée. J'ai maintenant trouvé le titre en français: Entre-lieu, parce que c'est vraiment entre deux choses.

On voit ici la gravure et une application à l'architecture à partir de la plaque de bois que j'ai coulée en aluminium en vue d'un projet 
d'intégration à un lieu. J'ai plusieurs réalisations dont celle-ci, pour moi très importante, qui a été faite avec l'architecte Paul Faucher. C'est à l'île du Moulin, à Terrebonne que j'ai appliqué le concept de l'Entre-lieu. D'un côté, la plaque a été suspendue au plafond et, de l'autre, l'impression. Cela demandait une collaboration entre l'artiste et l'architecte, une connaissance du lieu et du thème. Cet ancien moulin est aujourd'hui devenu une bibliothèque. J'ai fait un lien entre l'écriture et la gravure sur bois (qui est la première sorte d'écriture que les hommes ont inventée pour communiquer) et en même temps je retrouvais le cercle des grandes roues motrices de l'ancien moulin à scie. Les motifs sont des motifs aquatiques. On ne sait pas, bien sûr, si c'est l'architecte qui a fait la murale ou si c'est moi. Voilà un projet d'intégration art-architecture qui, je crois, est tout à fait réussi.

En 1984, j'ai tenu à Montréal cette exposition qui s'intitule Between. Voici une des réalisations grand format ( 8 pieds $x 8$ pieds). D'un côté, la plaque et, de l'autre, une impression rehaussée de couleurs. C'est également l'occasion, dans mon travail, du retour important, énorme de l'influence mexicaine et de l'art précolombien. Il y a comme un mélange des formes du territoire, de la géologie et des écritures précolombiennes.

Voici une autre grande pièce (mêmes dimensions), une gravure: au centre, la plaque gravée et, de chaque côté, les impressions sur toile.

Puis une autre oeuvre tirée de l'exposition Between: une évocation, sur le plan du relief, du nord d'un côté et du sud de l'autre. Les deux pôles des influences qui m'ont le plus marqué.

Ici, j'ai repris les plaques et les ai réinsérées dans le paysage pour leur donner un lieu. C'est dans un «pit» de gravier qui est à côté de chez moi. Les gens du village, en voyant ça, m'ont dit: "on sait immédiatement où tu prends tes choses, maintenant»!

Depuis quelques années, le passage est devenu plus important que le lieu:

à l'instant du passage

à l'instant d'être ailleurs

à l'instant du devenir

et de s'autogérer génétiquement.

C'est un état angoissant, l'entre-lieu: savoir qu'à l'instant d'ouvrir la porte ou de traverser le miroir, il y a le vide imaginé. Le lieu se crée, et disparaît à nouveau. Apatride et toujours de passage, emportant son lieu avec soi, se sentir l'observateur en mouvement, observant des lieux en changement. 


\section{POST-SCRIPTUM}

\section{4 juin 1987 - Aéroport J.F. Kennedy - New-York}

9:10 p.m. - Lieu de réflexion

Suis-je dans un musée? une église? un couvent?

Dans les jours de montage de l'exposition précédant le colloque, nous étions déjà dans un lieu habité.

EST-CE? L'énergie du lieu qui donna à mon équipe de montage, Johanne Roy, Pierre Leblanc, Jean-Michel Corrieo, cette solidarité d'oeuvrer dans la magie des lieux.

EST-CE? L'esprit des lieux intérieurs au musée qui nous motiva.

EST-CE? L'esprit intérieur du personnel du musée qui nous toucha.

EST-CE? Les attentes du lieu extérieur, conférenciers, artistes, étudiants, etc. qui nous "énergisa».

On n'en finira jamais d'interroger ce phénomène intérieur/extérieur. Je le crois maintenant, avec une journée de recul de l'événement "L'Esprit des lieux", alors que j'écris cette réflexion dans un lieu des plus éclatés, sans mémoire, l'ENTRE-LIEU de l'aéroport J.F. Kennedy, New-York, dans l'attente de mon prochain départ pour l'Islande.

Je constate que je fredonne encore les dernières chansons du souper de fermeture de "L'Esprit des lieux".

Actuellement, autour de moi, des Hindous, des Argentins, des Mexicains, des Japonais, des dizaines de nationalités se côtoient et se préparent à franchir, encore une fois, l'entre-lieu du retour ou du départ. Une évidence m'apparaît: ces milliers de lieux intérieurs qui circulent dans cet aéroport sont leurs propres lieux en déplacement dans le temps et l'espace avec sûrement une nostalgie du retour à leurs lieux d'origine qui leur donnent identités et énergie pour leurs déplacements dans l'espace.

L'heure du départ est arrivée.

Vol 614 - Direction Reykjavik, Islande. Je rentre à l'intérieur du 747 qui m'amènera au soleil de minuit.

"L'esprit des lieux", le colloque, les expositions et tous ces échanges de conférenciers se sont intériorisés dans ma mémoire.

Je pars avec tout cela. 
On n'en finira jamais d'interroger ce phénomène intérieur/extérieur. Je le crois maintenant, avec une journée de recul de l'événement "L'Esprit des lieux", alors que j'écris cette réflexion dans un lieu des plus éclatés, sans mémoire, l'ENTRE-LIEU de l'aéroport J.F. Kennedy, New-York, dans l'attente de mon prochain départ pour l'Islande. Je constate que je fredonne encore les dernières chansons du souper de fermeture de «L'Esprit des lieux».

Act:iellement, autour de moi, des Hindous, des Argentins, des Mexicains, des Japonais, des dizaines de nationalités se côtoient et se préparent à franchir, encore une fois, l'entre-lieu du retour ou du départ. Une évidence m'apparaît: ces milliers de lieux intérieurs qui circulent dans cet aéroport sont leurs propres lieux en déplacement dans le temps et l'espace avec sûrement une nostalgie du retour à leurs lieux d'origine qui leur donnent identités et énergie pour leurs déplacements dans l'espace.

L'heure du départ est arrivée.

Vol 614 - Direction Reykjavik, Islande. Je rentre à l'intérieur du 747 qui m'amènera au soleil de minuit.

"L'esprit des lieux", le colloque, les expositions et tous ces échanges de conférenciers se sont intériorisés dans ma mémoire.

Je pars avec tout cela.

\section{5 juin 1987 - Reykjavik, Islande $11: 30$ p.m.}

A l'approche du soleil de minuit.

Me voilà en terre nordique, lieu de glaciers, de geysers, de lumières et de canards montés au Nord.

Pourquoi j'y suis?

\section{7 juin 1987 - Reykjavik, Islande 2:00 a.m.}

Le parc de Reykjavik est animé d'une vie nocturne au soleil de minuit. Les canards de Morrisseaunord survolent lé lac du centre-ville. Des milliers d'insectes se jouent de courbes sans fin, nourrissent les oiseaux et disparaissent. 
C'est la nuit sans la nuit avec l'énergie solaire-lumière.

\section{2 juin 1987 - Reykjavik New-York}

Icelandair Vol 615 via New-York.

Je survole le Groenland et les terres neuves. Le soleil nous suit, le temps est arrêté et l'espace se déplace.... toujours à la même heure.

J'arrive à New-York. Direction Greenwich, au Sweet Basil Jazz Bar de Cedar Walton, Ron Carter, Billy Higgins. Jazz des dimanches aprèsmidis dans un New-York ensoleillé du même soleil islandais. Le salut des grands nobles du jazz noir. New-York est là dans son délire et son agression de vie primaire.

Carolina photographie les notes à la seconde pour les révéler dans sa chambre noire de Londres.

\section{3 juin 1987 - New-York}

Ce matin au Time Square - quel beau square, du temps à passer, à créer...

A cet endroit, ce matin, à 11:00, il fut attaqué par quatre personnes un incident. Le soir me semble plus de sécurité puisque, hier tard, je me promenais entre la 42ième et Time Square.

Le cirque de nuit était là, ouvert à tous. C'était beau, doux et calme. Nous suivions les vagues des vibrations de New-York comme les insectes du parc de Reykjavik.

La mémoire des lieux se mêle de souvenirs, d'actions/séductions.

\section{4 juin 1987 - Aéroport La Guardia, New-York 7:40 p.m.}

Le vol via Montréal m'emporte du Sud au Nord. Réchauffé de lieux multiples vécus depuis le départ de Val-David via Rimouski un 26 mai. La magie se poursuit et m'habite.

New-York - Entre-lieu - Montréal: nous nous préparons à partir. Entre-temps: un vol lève dans «l'apesanteur sans fiction». Vol 742 Eastern arrive à New-York. 
Trans World s'envole vers?...

Pan Am est sur la piste...

TWA est en changement...

American Airline arrive au terminus...

Dans cette musique d'énergie et de désir de partir, les sons du saxophone me rappellent le village - l'autre - l'entre-lieu, comme la présence de l'ami brésilien me rappelle les plages de Rio, en mémoire l'instant des plages de Janeiro et des banlieues de Londres de Carolina photographiant le jazzman du village new-yorkais.

\section{4 juin 1987 - Val-David, P.Q.}

\section{1:30 p.m. - Rang de la Montée Gagnon}

J'arrive et les feuilles sont nées malgré moi.

La nature a poussé.

La nature est!

Les grandes pluies des débuts d'été.

J'y suis et le lieu est - a été - et sera...

J'arrive en pleine abondance de la nature. Je ne fais qu'arriver en ce lieu qui est un lieu.

Les marguerites, les lupins, les herbes du printemps rouge sont. Les échographies du printemps sont passées à l'été. 


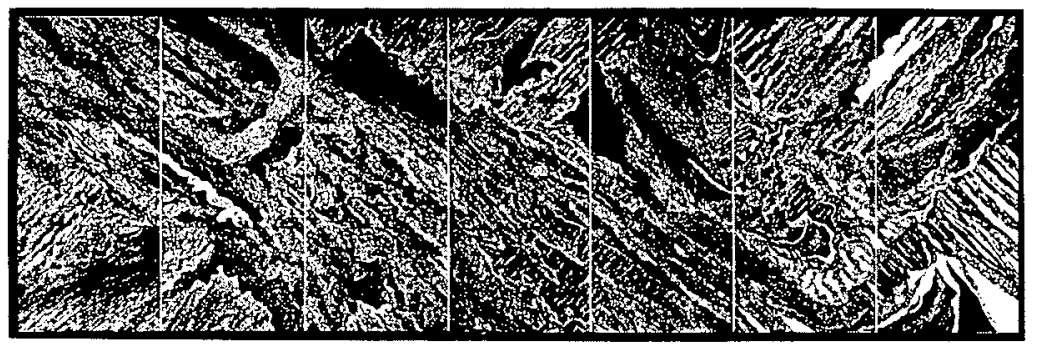

Gravure sur bois, $228 \times 707 \mathrm{~cm}, 1979-80$

(photo: Centre de documentation Yvan Boulerice)

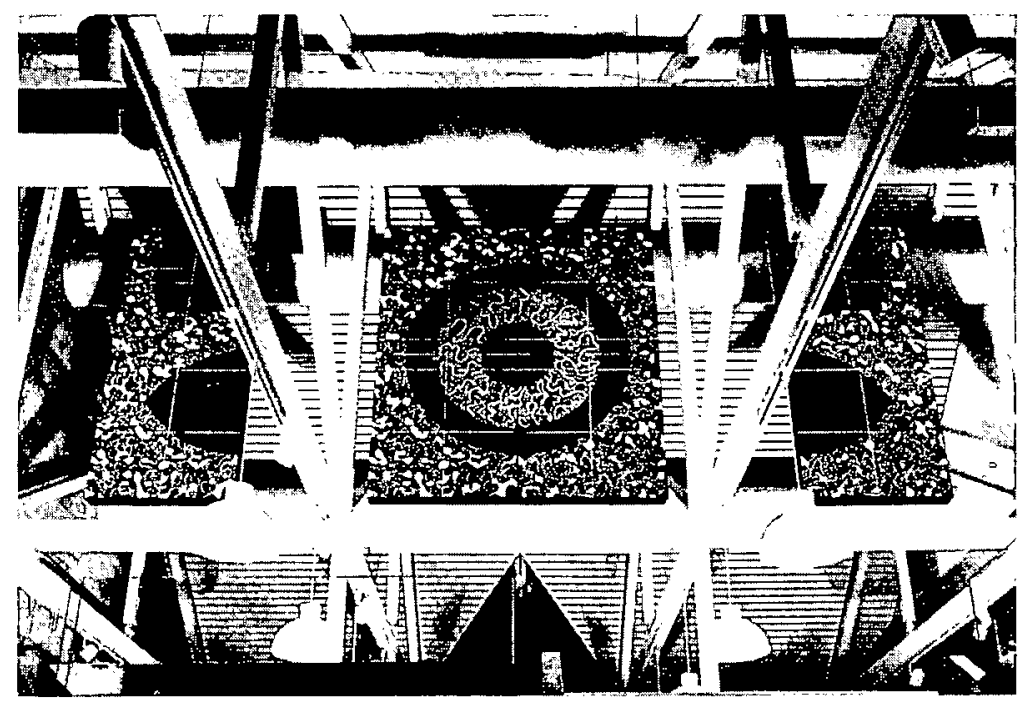

Collaboration avec Paul Faucher, architecte

Gravures murales, impression sur toile de la série Between

Ile du Moulin, Terrebonne, 1983-84 


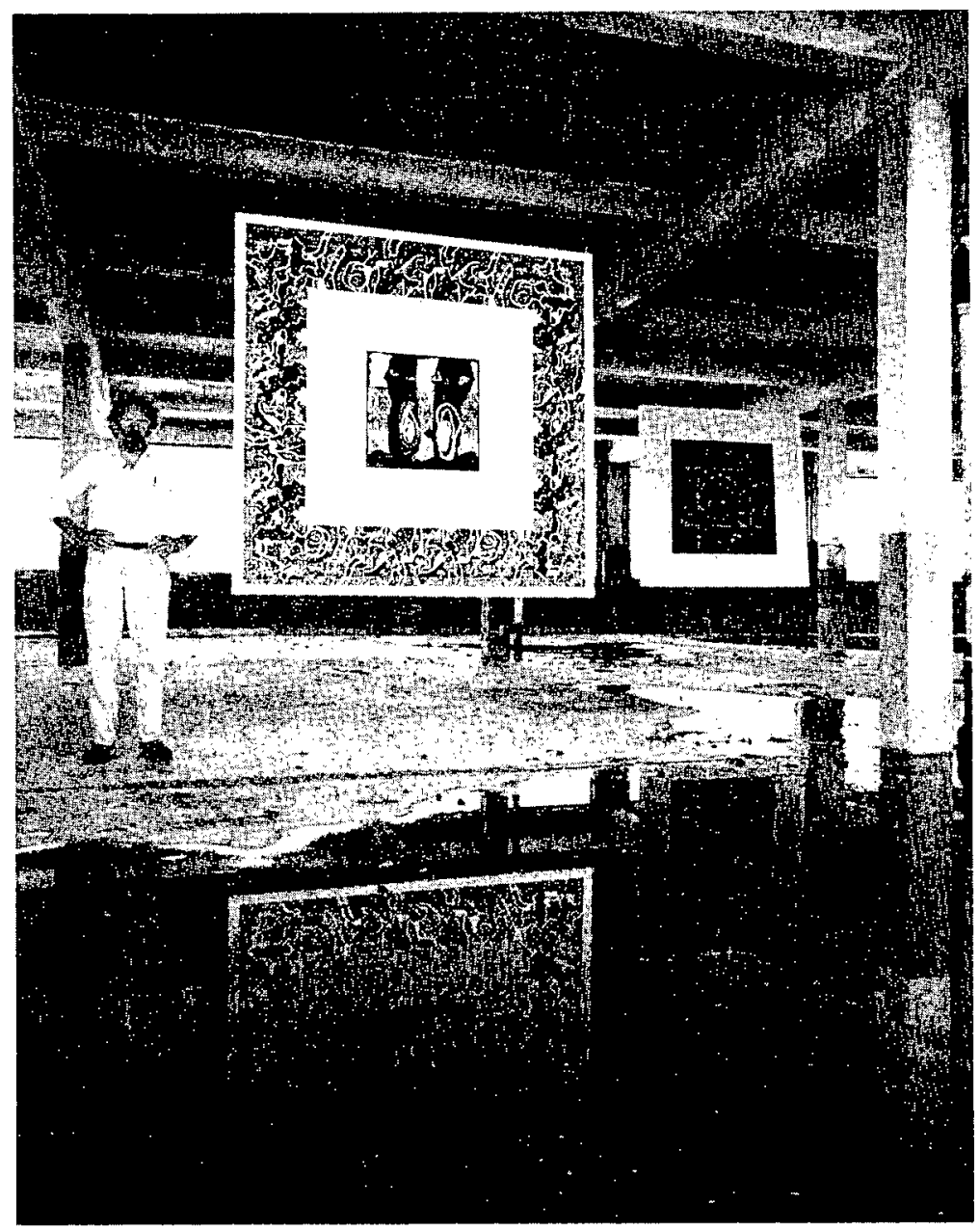

Gravure du bois, $226 \times 226 \mathrm{~cm}, 1986$

Installation usine Tricofil, Lieu de pouvoir. (photo: Lucien Lisabelle) 


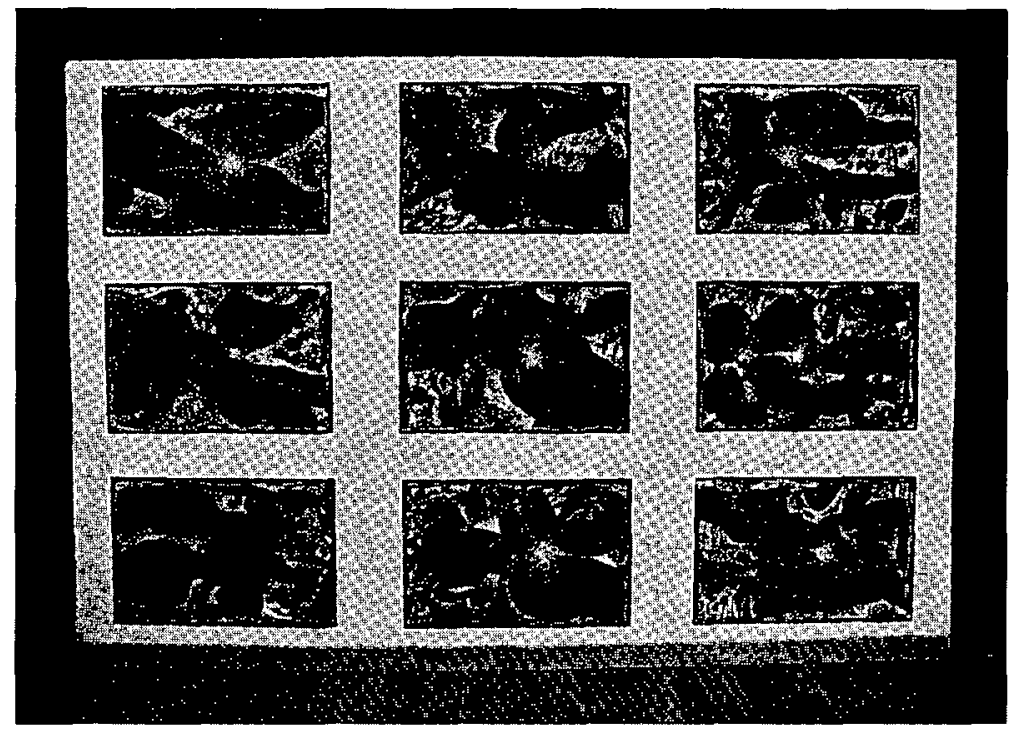

Installation, gravures sur bois, $243 \times 366 \mathrm{~cm}, 1987$

Musée régional de Rimouski

(photo: Gérald Bouillon)

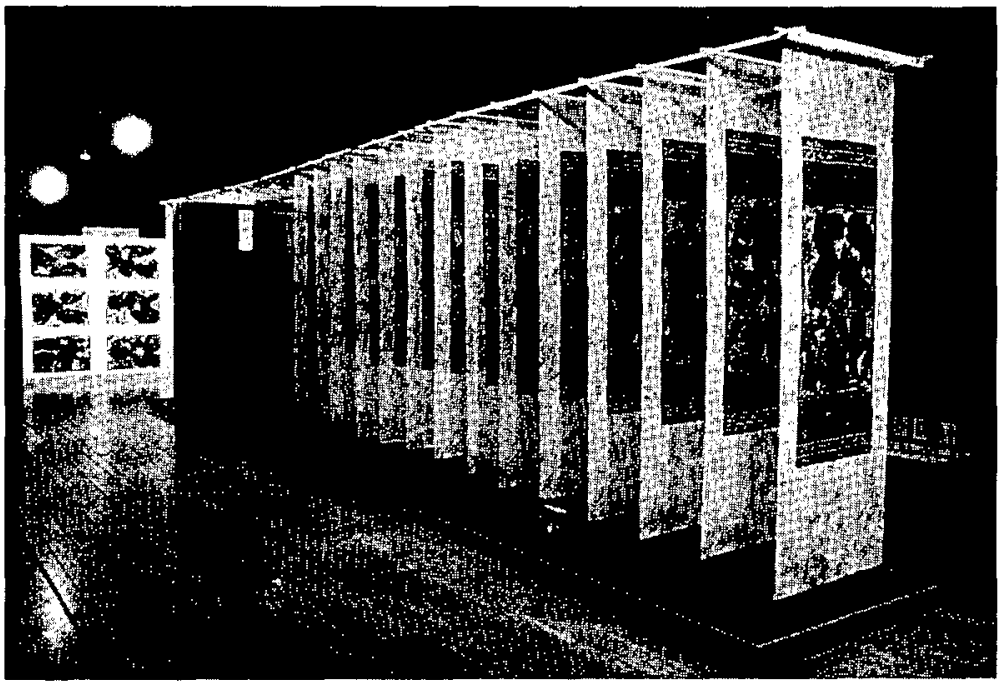

Échographies du printemps, installation, gravures, 1987

Musée régional de Rimouski

(photo: Gérald Bouillon) 